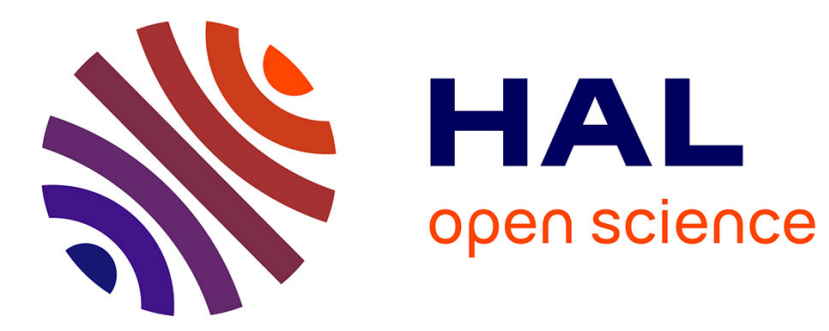

\title{
L'US Forest Service et la biodiversité
}

Sally Collins, Hutch Brown

\section{- To cite this version:}

Sally Collins, Hutch Brown. L'US Forest Service et la biodiversité. Revue forestière française, 2011, 63 (5), pp.641-649. 10.4267/2042/46110 . hal-03449692

\section{HAL Id: hal-03449692 \\ https://hal.science/hal-03449692}

Submitted on 25 Nov 2021

HAL is a multi-disciplinary open access archive for the deposit and dissemination of scientific research documents, whether they are published or not. The documents may come from teaching and research institutions in France or abroad, or from public or private research centers.
L'archive ouverte pluridisciplinaire HAL, est destinée au dépôt et à la diffusion de documents scientifiques de niveau recherche, publiés ou non, émanant des établissements d'enseignement et de recherche français ou étrangers, des laboratoires publics ou privés. 


\title{
L'US Forest Service et La biodiversité
}

\author{
Sally Collins - Hutch Brown
}

L'US Forest Service ${ }^{(1)}$ a été créé en 1905, durant une période marquée par une déforestation galopante aux États-Unis. En réaction à la perspective d'une pénurie de bois mais aussi aux inondations catastrophiques, à l'érosion des sols et à l'importance des matières en suspension dans les cours d'eau, dues à la déforestation, le Congrès des États-Unis donna son aval en 1897 à un système de réserves forestières fédérales destinées à " améliorer et protéger la forêt dans ses limites actuelles, à maintenir les conditions d'un écoulement favorable des cours d'eau, ainsi qu'à garantir un approvisionnement régulier en bois " (Organic Administration Act). Le dispositif national forestier protège désormais 77,164 millions d'hectares de forêts nationales réparties sur tout le territoire des États-Unis (8,5\% du territoire national), et l'US Forest Service a la responsabilité de les gérer durablement. Bien que la mission première et permanente de l'US Forest Service ait toujours été la conservation de ces forêts, son objectif a évolué avec le temps, notamment afin de répondre à une demande publique qui s'est déplacée, prenant en compte les préoccupations du public relatives à la perte de biodiversité.

\section{UN CGUR DE MISSION EN PLEINE ÉVOLUTION}

Dès sa création, l'US Forest Service s'est pleinement investi dans la protection des forêts, des bassins versants et des habitats naturels, essentiellement au profit du grand gibier. Les premiers forestiers étaient armés, notamment pour tirer les ours, les pumas et autres grands prédateurs. Aldo Leopold qui passa la première partie de sa carrière à l'US Forest Service fut un jour amené à ouvrir le feu sur une meute de loups : " J'étais alors jeune, impulsif et irréfléchi ; sachant que moins de loups signifiait plus de cervidés, je pensais qu'un monde sans loup serait le paradis des chasseurs. " (Leopold, 1949). Les populations locales étaient alors clairement en faveur de l'extermination des prédateurs, surtout pour protéger les troupeaux. Dans les années 1940, la plupart des grands prédateurs avaient été éliminés de la majeure partie de leur aire de répartition.

C'est alors que Leopold prit conscience de son erreur. En tant que fondateur et pionnier de la gestion scientifique de la faune sauvage aux États-Unis, Leopold comprit le rôle que les prédateurs jouaient dans le fonctionnement des écosystèmes, et par conséquent la catastrophe écologique qui résulterait de leur disparition. Dans le contexte qui suivit la fin de la Seconde Guerre mondiale, l'US Forest Service mit l'accent sur la production et la récolte intensive de bois pour répondre à une demande en logements qui explosait. Or, de son étude des forêts allemandes gérées pour la production de bois, Leopold avait retenu qu'un tel type de gestion couplé avec la disparition des grands prédateurs pouvait conduire à un réel appauvrissement biologique (Leopold, 1936). Il avait également compris qu'il faudrait une gestion très volontariste pour

(1) L'US Department of Agriculture (USDA) assure la tutelle (mais pas exactement au sens français) de l'US Forest Service (USFS) qui est une agence du gouvernement. L'USFS dispose d'une organisation à 4 niveaux hiérarchiques et géographiques : 5 directions spécialisées qui assistent le chef de l'USFS, 9 grandes interrégions, des forest supervisors (compétents pour une ou plusieurs forêts nationales) et environ 500 unités territoriales de base : les Ranger districts (note du traducteur). 
revenir ensuite à des écosystèmes en bonne santé : "sauvegarder chaque boulon et chaque écrou doit être la première précaution d'un bricoleur intelligent ${ }^{(2)}$ » (Leopold, 1953).

La polarisation de l'US Forest Service de l'après-guerre sur le bois conduisit à de premiers affrontements dès qu'un nombre significatif de citoyens commencèrent à savoir ce qu'ils attendaient exactement des espaces publics qu'ils considéraient comme leur propriété. La croissance du niveau de vie et l'augmentation du temps dévolu aux loisirs, couplées avec une nouvelle mobilité permise par les voitures et les routes en forêt, conduisirent à un nombre toujours plus grand de visiteurs dans les forêts fédérales. Ils venaient notamment pour découvrir des paysages grandioses, et beaucoup d'entre eux étaient choqués de constater l'ampleur des traces d'une exploitation industrielle du bois. Les écrits d'Aldo Leopold et le livre Printemps silencieux de Rachel Carson (1962) qui mettait en garde sur les effets des produits chimiques largement épandus sur les oiseaux, aidèrent grandement à sensibiliser les citoyens américains aux problèmes de la faune sauvage. De surcroît le niveau croissant d'éducation du public, de plus en plus méfiant visà-vis des arguments fondés sur la seule expertise professionnelle des gestionnaires, conduisit à la montée des revendications en faveur d'une plus grande association du public dans la gestion des terrains domaniaux (Cortner, 2003 ; Gibbons, 1999).

En réaction, le Congrès des États-Unis adopta des lois cherchant d'une part à trouver un équilibre entre la satisfaction des besoins en bois et les autres usages des forêts fédérales, et d'autre part à donner au public des occasions de peser sur la gestion forestière. En particulier, la loi sur les espèces en danger de 1973 mettait en demeure l'US Forest Service de garantir la protection des habitats nécessaires aux espèces menacées ou en danger. La loi forestière de 1976 exigeait que chaque forêt fédérale ou chaque territoire pastoral fédéral dispose d'un plan de gestion comprenant des orientations visant à la diversité des associations végétales et communautés animales (USDA Forest Service, 1993), au terme d'une procédure offrant au public un certain nombre d'occasions pour apporter des contributions. Pour aider les unités à concevoir leur plan de gestion, l'US Forest Service définit en 1982 une procédure à suivre formellement. Cette règle de planification imposait à chaque unité de gestion " de garantir à la faune et à la flore des habitats permettant de préserver un niveau de population viable des espèces de vertébrés indigènes. " (USDA Forest Service, 1982).

Le nouveau cadre juridique et procédural avait été conçu, au moins en partie, pour réduire les litiges portant sur la gestion des forêts nationales (Gippert et DeWitte, 1990). Mais au lieu de rapprocher les parties prenantes, il devint un nouveau terrain de bataille pour les défenseurs de l'environnement et pour l'industrie du bois. Dans les années 1980 et 1990, la biodiversité devint le mot d'ordre d'une des thèses qui s'affrontaient. Les nouveaux textes législatifs et réglementaires qui incluaient la clause des "populations viables » étaient largement utilisés pour bloquer les projets de l'US Forest Service au nom de la protection de la biodiversité. L'illustration la plus célèbre en est sans doute la controverse de la Chouette tachetée.

La Chouette tachetée (Strix occidentalis) a pour habitat les gigantesques arbres pluri-centenaires des régions occidentales du Nord de la Californie, de l'Oregon et de l'État de Washington. En 1990, cette chouette avait été classée dans la liste des espèces menacées, dans ce qui était alors la zone la plus importante pour la production de bois sur l'ensemble du territoire américain. Les défenseurs de l'environnement, en opposition frontale avec les populations locales dont l'économie dépendait du bois, mirent l'US Forest Service au tribunal afin de bloquer toutes les ventes de bois dont l'exploitation était susceptible d'affecter l'habitat de la Chouette tachetée. En 1994, le président Clinton prit position à travers le Plan forestier pour la région Pacifique-Nord-Ouest, qui mit en réserves intégrales les peuplements ayant atteint leur stade ultime.

(2) Libre traduction de "to keep every cog and wheel is the first precaution of intelligent tinkering" (note du traducteur). 


\section{ÉMERGENCE D’UNE APPROCHE ÉCOSYSTÉMIQUE}

Les autres territoires forestiers restèrent ouverts à la gestion et à l'exploitation forestière, mais cette décision présidentielle marqua la fin des coupes de bois à grande échelle dans les forêts nationales. En 1990, la récolte de bois chuta, après avoir atteint le niveau record de 30 millions de $\mathrm{m}^{3}$ en 1987, pour revenir au niveau d'avant-guerre. Les récoltes annuelles se sont depuis lors stabilisées aux environs de 5 millions de $\mathrm{m}^{3}$ par an, principalement suite à des interventions justifiées par d'autres considérations, comme des coupes sanitaires. Pourtant la controverse a perduré, mettant au second plan des menaces autrement plus graves pour la santé des forêts américaines :

- La première menace pour la Chouette tachetée est désormais la compétition avec la Chouette rayée (Strix varia), une espèce invasive originaire de l'Est des États-Unis. Des insectes invasifs et des maladies menacent une série d'essences forestières depuis le Frêne jusqu'au Châtaignier, au Thuya et aux Pins blancs. Des dizaines de millions d'hectares de terrains pastoraux ont été dégradés par des espèces herbacées invasives, comme le Brome des toits (3);

- Le changement climatique a contribué à accentuer les sécheresses dans certaines zones, en même temps que les incendies, en surface et en intensité : plus de 28000 habitations, installations industrielles ou dépendances ont été détruites par des incendies de forêts ou de friches. Le changement climatique a également favorisé l'extension géographique et le niveau des populations des insectes sous-corticoles, qui ont tué des peuplements de pins et d'autres types de forêts sur de vastes surfaces ;

- En raison de la croissance démographique, l'artificialisation des sols menace les forêts privées. À titre d'exemple, les prévisions affichent l'urbanisation inéluctable de 23 millions d'hectares de forêts, soit l'équivalent de la surface du Portugal (Stein et al., 2009).

Depuis les années 1980, chaque nouveau chef de l'US Forest Service a reconnu le besoin d'une approche équilibrée qui prenne en compte ce que la science peut dire, ce que les décideurs politiques veulent, ce que le public attend, et ce qui est nécessaire pour faire face aux défis du $X X I^{e}$ siècle. Pour y répondre, l'US Forest Service a dû complètement se réinventer et ce faisant, c'est toute la profession forestière qui a évolué avec lui aux États-Unis.

La gestion traditionnelle multifonctionnelle est ciblée sur la fourniture durable de biens et services, dont entre autres le bois, l'eau, la faune sauvage, les loisirs de plein air et beaucoup d'autres biens et services encore. Dans les années 1980, cette approche (avec son tropisme fort en faveur de la production de bois) prévalait au sein de l'US Forest Service, mais ce n'est aujourd'hui plus le cas (Bosworth et Brown, 2007a, 2007b). Actuellement, la polarisation se fait sur la nécessité de garantir sur le long terme l'intégrité de l'écosystème, en utilisant la sylviculture pour atteindre, à l'échelle des paysages, un état restauré sur les plans de la santé et de la résilience, et ceci aussi bien pour les forêts que pour les terrains pastoraux. La polarisation sur des objectifs à long terme, y compris en termes de biodiversité, est la nouvelle norme, une nouvelle manière de faire son métier de forestier. Les acteurs de la forêt publique comme de la forêt privée ont généralement adopté cette nouvelle manière de voir les choses, et les programmes de formation universitaire ont été au rendez-vous, avec des cursus portant sur l'approche intégrée des ressources naturelles dans tous les domaines, alimentant ainsi le secteur forestier avec des nouvelles personnes ressources, disposant de compétences et d'une vision nouvelles. Un nouveau langage professionnel est en train d'émerger, autour de nouvelles notions comme celle de restauration d'écosystèmes. Ce langage reflète - et engendre - une profonde mutation culturelle (Collins et Brown, 2008).

(3) Bromus tectorum, une Poacée, est originaire des régions tempérées de l'ancien monde : Europe, Afrique du Nord, îles Canaries, Asie occidentale et centrale (de la Turquie à l'Afghanistan), orientale (Chine, Sibérie), ainsi que du sous-continent indien. Elle s'est naturalisée en Amérique du Nord et du Sud ainsi qu'en Australie. C'est une plante qui affectionne les terrains secs et calcaires (note du traducteur, à partir de Wikipedia consultée le 15 mai 2011). 


\section{L'ÉVOLUTION DÉCOULANT D'UN RAISONNEMENT EN TERMES DE SERVICES ÉCOSYSTÉMIQUES}

Nous sommes convaincus que la politique forestière américaine, conduite par l'US Forest Service, a évolué dans le bon sens. Et pourtant des questions redoutables subsistent. Une première interrogation porte sur le cadre réglementaire s'appliquant à la protection des espèces vivant dans les forêts et les terrains pastoraux. Dans un contexte de changement climatique, jusqu'à quel point est-il réaliste de chercher à gérer les forêts au profit des populations viables d'espèces existantes ? La diversité est certainement une réponse nécessaire. Étant donné les incertitudes liées au changement climatique, la stratégie de l'US Forest Service vise désormais à maximiser la diversité des communautés végétales et animales à l'échelle du paysage pour minimiser le risque (USDA Forest Service, 2010). Conscient du risque et des incertitudes, l'US Forest Service a travaillé avec ses partenaires et avec le public pour élaborer de nouvelles règles de gestion et de planification forestière (USDA Forest Service, 2011), qui prennent en compte une large gamme de biens matériels et immatériels et de services auxquels une valeur est reconnue, au travers d'une approche écosystémique et participative, notamment en maximisant la diversité à l'échelle du paysage.

Quoi qu'il en soit, mandater le gestionnaire forestier pour assurer la protection d'espèces particulières dans des sites où elles ne peuvent plus être considérées comme viables apparaît comme une solution à courte vue et inconsidérée. Plus encore, c'est focaliser l'attention sur la gestion des espaces protégés, une stratégie de conservation de la biodiversité qui laisse de plus en plus dubitatif. Les terrains fédéraux sont un refuge précieux pour des espèces rares et vulnérables (Groves et al., 2000), mais leur capacité à garantir la durabilité des communautés indigènes végétales et animales est limitée. Comme Aldo Leopold le rappelait, "le gouvernement ne peut pas se porter acquéreur de n'importe quel terrain ; les propriétaires fonciers privés méritent d'être pris en considération, dans tous les sens du terme (4) " (Leopold, 1935). Seulement $20 \%$ des terrains boisés relèvent des forêts nationales, alors que $56 \%$ sont dans les mains des propriétaires privés ${ }^{(5)}$, et dans l'Est des États-Unis ce dernier pourcentage s'élève à près de $83 \%$ (Smith et al., 2004). Les paysages forestiers américains sont habituellement pris en étau entre les gestionnaires publics et privés. Dans beaucoup de zones de l'Ouest des États-Unis, les sommets sont couverts par des forêts nationales, les pentes sont aussi des terrains publics, mais gérés par l'US Bureau of Land Management, alors que les terrains plats et les fonds de vallées bien alimentés en eau sont privés. Un seul gestionnaire maîtrise rarement un bassin versant tout entier, et les menaces comme les incendies de forêt ou les espèces invasives méconnaissent les limites de propriété. Le défi est donc de planifier et d'agir de façon concertée, à une échelle géographique pertinente, sans s'arrêter aux limites de propriété, pour protéger la biodiversité, la qualité de l'eau et tout ce qui est pertinent du point de vue écologique.

Au lieu de rapprocher des gens qui devraient collaborer, la protection de la biodiversité fondée sur une approche espèce par espèce les a divisés. Les gens ont naturellement tendance à protéger ce qui a de la valeur à leurs yeux, et ils reconnaissent de la valeur à ce qu'ils peuvent utiliser pour améliorer leur bien-être personnel. En se polarisant sur le maintien de populations animales viables dans les espaces protégés, l'approche espèce par espèce a relégué au second plan toute la gamme des bénéfices que les populations tirent des forêts et des pâturages, tant publics que privés, laissant en de nombreux endroits du pays un lourd passif d'amertume, de conflits et de méfiance. Des opportunités de construire une gouvernance collaborative ont dès

(4) Libre traduction de The government cannot buy "everywhere". The private landowner must enter the picture (note du traducteur). (5) Le reste (environ $24 \%$ ) relève d'autres agences fédérales (comme le Service des parcs nationaux et le Bureau of Land Management) et dans une moindre mesure des États fédérés (note du traducteur). 
lors été manquées, alors qu'il aurait été possible de mobiliser les citoyens américains autour du défi de la gestion des terrains publics qu'ils considèrent comme leur propriété. L'opportunité d'attirer l'attention sur l'enjeu de dépasser les limites de propriété pour relever ces défis a également été perdue, notamment pour lutter contre la perte des habitats naturels dans les forêts privées.

Durant le mandat de Dale Bosworth (directeur de 2001 à 2007), l'US Forest Service adopta une tout autre stratégie : une approche en termes de services écosystémiques (Collins et Larry, 2007). Les services écosystémiques provenant de la forêt comprennent des services variés, comme des services d'auto-entretien (formation des sols et production photosynthétique primaire), des services d'approvisionnement (bois et eau), des services de régulation (pollinisation et débits des cours d'eau) et des services culturels (émotion esthétique et loisirs de plein air) ${ }^{(6)}$. Une approche en termes de services écosystémiques a plusieurs avantages :

- Elle place les gens au cœur des politiques de conservation. Du point de vue des services écosystémiques, la fonctionnalité des écosystèmes est le support "vivant " de services appréciés par les hommes. Les activités de gestion sont alors conçues pour maintenir ou renforcer ces services, parce que ces derniers correspondent aux besoins et attentes du public et non parce qu'ils sont une composante d'une notion suspecte de nature originelle.

- Elle stimule la coopération au-delà des limites de propriété. Une approche en termes de services écosystémiques légitime une large gamme d'attentes et de besoins, et organise la gestion des ressources naturelles autour d'eux. Dans le respect de chacun, les parties prenantes peuvent joindre leurs efforts au sein d'un paysage vécu comme partagé, formuler des objectifs communs et se mettre d'accord sur la meilleure manière de les atteindre.

- Elle prend en compte et valorise le changement. Du point de vue des services écosystémiques, les besoins humains augmentent, et leurs tenants et aboutissants historiques évoluent. Les gestionnaires forestiers mettent l'accent sur le maintien et le renforcement de la capacité des paysages à s'adapter au changement tout en continuant à fournir les services écosystémiques. Un écosystème restauré peut ne pas ressembler au paysage originel, mais il doit être un " système » en bonne santé et résilient, capable de satisfaire les besoins de la société avec une large gamme de services écosystémiques.

- Elle prend en compte et valorise à leur juste prix les écosystèmes. Du point de vue des services écosystémiques, les paysages sont le vrai capital naturel productif. Les gestionnaires forestiers prennent en compte et valorisent les « immobilisations » dont ils s'occupent, mesurant les flux et les stocks dans les services écosystémiques et s'assurant que ceux qui leur font confiance sont bien au courant de leur valeur et du coût de leur perte.

Un expert a chiffré la valeur du dispositif des forêts nationales à 800 milliards de dollars, sur la base de ce qu'apportent chaque année à la société les services écosystémiques. L'US Forest Service mobilise le budget ${ }^{(7)}$ que lui alloue le Congrès des États-Unis, certains subsides complémentaires de ses partenaires, et dans une nettement moindre mesure les droits d'entrée et la

\footnotetext{
(6) Cf. le Millennium Ecosystem Assessment (MEA, 2005) (note des auteurs).

(7) Le budget de l'USFS en 2011 est d'environ 5,1 milliards de dollars (en réduction de 178 millions de dollars par rapport à 2010), voté par le Parlement par programmes limitatifs : gestion des forêts nationales, recherche, prévention et lutte contre les incendies dans les forêts nationales, assistance technique et financière à la forêt privée, programme Forest Legacy... La fongibilité entre programmes doit faire l'objet d'un nouveau vote du Congrès, qui peut intervenir en urgence, par exemple si l'ampleur des feux de forêts exige un redéploiement budgétaire. À ce budget voté s'ajoutent :

- environ 200 millions de dollars venant d'un pourcentage sur les ventes de bois (dont le produit est ventilé entre le Trésor public à raison de $75 \%$ et les comtés à raison de $25 \%$ ) et qui sont affectés au reboisement des coupes ;

- des sommes variables provenant des droits d'accès qu'une loi de 2002 a institués pour certains sites touristiques aménagés, et qui relèvent d'une régie locale garantissant aux citoyens contributeurs que les droits perçus dans un site donné restent intégralement affectés à l'aménagement et au fonctionnement du site ;

- la force de travail venant des quelque 8 ooo volontaires qui se proposent chaque année de travailler gratuitement à l’USFS pour une période minimale de 3 mois, 3 jours par semaine (après une semaine de formation), essentiellement pour l'accueil estival dans les forêts nationales, les musées de l'USFS et les boutiques de l'USFS (note du traducteur).
} 
vente de ses produits. Cela permet à l'État d'investir dans le capital naturel productif dans l'objectif de fournir des services écosystémiques à partir des forêts nationales et des terrains pastoraux qu'il gère : eau de qualité, sols en bon état, faune sauvage et bien d'autres choses encore. En agissant ainsi, le service forestier assume la responsabilité traditionnelle des pouvoirs publics de fournir des biens publics à partir des terrains publics.

En forêt privée, les opportunités pour investir de cette manière dans le capital naturel productif sont cependant moindres, parce que peu de services écosystémiques se voient reconnaître de la valeur par le marché. Chaque jour, environ 2400 ha de terrains naturels sont artificialisés aux États-Unis, parce que leur principale valeur marchande est leur valeur foncière. Les agences gouvernementales et les ONG peuvent apporter leur concours aux propriétaires forestiers via les " servitudes contractuelles environnementales ${ }^{(8)}$ " ou en leur fournissant sous diverses formes une assistance financière ou technique, mais ce n'est clairement pas à la hauteur de l'enjeu. Les États-Unis ne peuvent pas compter seulement sur les aides gouvernementales ou sur la bonne volonté des mécènes et des propriétaires privés.

Du point de vue des services écosystémiques, il est possible de stimuler une croissance économique intelligente en recourant à la puissance des mécanismes du marché. En recourant prudemment à des outils de marché, la valeur que la société attache aux services écosystémiques peut se transformer en argent sonnant et trébuchant. Une première voie passe par les dispositifs d'écocertification qui misent sur la prédisposition de certains consommateurs à payer un supplément pour avoir accès à du bois issu de forêts gérées durablement. Une autre voie passe par des dispositifs mettant sur le marché des " crédits " qui bénéficient aux propriétaires pour fournir des services tels que la séquestration de carbone, la protection de la biodiversité, la restauration des zones humides ou la fourniture d'eau de qualité. En encourageant l'écocertification et l'émergence d'un marché de crédits, l'US Forest Service, en partenariat avec d'autres services du ministère de l'Agriculture (USDA), encourage la mise en œuvre de nouveaux moyens concourant à la conservation des forêts privées.

Les résultats de cet effort sont concrets. Depuis que l'US Forest Service s'est investi dans le marché des services écosystémiques, un certain nombre de projets ont été mis sur les rails. Malgré la faiblesse du budget que lui alloue le ministère de l’Agriculture, le service des marchés environnementaux a identifié trois terrains d'expérimentation pour la création de tels marchés :

- le partenariat Willamette (http://willamettepartnership.org/),

- le projet d'un marché de services environnementaux sur le bassin versant de la rivière Ohio (http://my.epri.com/portal/sever.pt?open=512\&objlD=423\&mode=2\&in_hi_userid=2\&cached=true),

- et la Banque de la Chesapeake Bay (http://www.pinchot.org/gp/bay_bank).

En parallèle, le ministère de l'Agriculture a initié le programme "Exploitations agricoles du futur ", au sein duquel les propriétaires exploitants investissent dans une gamme de marchés environnementaux (http://www.usda.gov/oce/environmental_markets/farm.htm/).

(8) Traduction proposée par la Société française du droit de l'environnement (SFDE) pour conservancy easement. Cette traduction n'est sans doute pas optimale, car cette terminologie a donné une connotation malheureuse de contrainte imposée. Par ailleurs il n'existe actuellement pas de marché des servitudes en droit français, et la très grande majorité des servitudes en France se situe entre un fonds dominant et un fonds dominé, ce qui n'est pas le cas pour les conservancy easements. Mais si l’on veut néanmoins employer la notion de servitude, il s'agit d'une " servitude de ne pas faire ", qui se définit au cas par cas en fonction d'un enjeu identifié par les deux parties : ne pas défricher, ne pas couper, ne pas drainer, etc. (note du traducteur).

Cet outil est un accord librement négocié entre un propriétaire foncier et une agence gouvernementale ou une ONG, par laquelle il est juridiquement établi une limitation aux options de mise en valeur de la propriété. L'accord peut être trouvé gratuitement ou bien donner lieu à une transaction financière ; il engage tous les propriétaires ultérieurs mais ne modifie en rien le reste des prérogatives s'attachant à la propriété privée. La surface couverte par de tels accords est relativement faible. Selon Butler et Leatherberry (2004), sur les 105 millions d'hectares de forêt privée familiale, seulement 4,9 millions d'hectares (soit moins de 5 \%) sont concernés par de tels accords. Quoi qu'il en soit, ces accords sont particulièrement destinés à protéger des forêts à forte valeur patrimoniale ou jouant un rôle écologique particulier, comme d'importants corridors écologiques. L'US Forest Service, grâce à son programme Forest Legacy, a financé l'achat de servitudes contractuelles environnementales sur 800 ooo ha répartis sur tout le pays (note des auteurs). 
Une autre approche très prometteuse a émergé dans le cadre d'un partenariat entre l'US Forest Service et l'Autorité du bassin de Denver, mobilisant un budget municipal pour gérer les forêts en tête du bassin versant dans l'objectif de fournir une eau de qualité à Denver (http://www.denverwater.org/SupplyPlanning/WaterSupply/PartnershipUSFS/). Les gestionnaires des forêts nationales travaillent avec les chercheurs de l'US Forest Service pour mieux quantifier la valeur des services écosystémiques provenant des forêts publiques (Smith et al., 2011). L'US Forest Service et l'ONG internationale Forest Trends (9) gèrent des sites internet où l'on peut trouver des informations sur les marchés et sur le paiement des services environnementaux (http://www.fs.fed.us/ecosystemservices/; http://www.forest-trends.org/program.php?id=69).

\section{CONCLUSIONS}

Pour relever ces défis, tout particulièrement dans un contexte de changement climatique et d'une forte croissance économique, les gestionnaires fonciers américains auront besoin d'adopter et de mettre en œuvre une approche en termes de services écosystémiques. Aldo Leopold le disait à sa manière : "La plus ancienne responsabilité humaine est celle de vivre sur un morceau de territoire sans le dilapider " (Leopold, 1938). Nous sommes convaincus que la même responsabilité sera au cœur des préoccupations du XXIe siècle en ce qui concerne les politiques de conservation. Il ne s'agira pas de chercher la protection des espèces une par une sur les terrains publics, mais de satisfaire une large gamme de besoins humains en mobilisant tous les territoires à cet effet, avec un accent mis sur les territoires de vie des populations, et en particulier les forêts privées. Nous sommes convaincus que des marchés environnementaux bien conçus et bien pilotés représentent un outil puissant de conservation (au sens large, incluant la biodiversité) pour les générations futures.

Sally COLLINS

Ingénieure forestière à la retraite

Directrice adjointe de l'US Forest Service de 2001 à 2008

Première directrice du service des marchés environnementaux

du ministère américain de l'Agriculture, de 2008 à 2010

Fellow, Rights and Resources Initiative

Natural Resources Consultant

602 Indian Lookout Road

LYONS, CO 80540-1919

(ÉTATS-UNIS D'AMÉRIQUE)

(scollins@rightsandresources.org)
Hutch BROWN

Conseiller en politique forestière

Chief's Office, $4 \mathrm{NW}$

USDA Forest Service

1400 Independence Ave. SW

Mailstop Code 1131

WASHINGTON, DC 20250-1131

(ÉTATS-UNIS D’AMÉRIQUE)

(hutchbrown@fs.fed.us)

Traduit de l'anglais américain par Christian Barthod

\footnotetext{
(9) Forest Trends est une ONG internationale basée à Washington, créée en 1999 par certains dirigeants d'organisations de protection de la nature, des industriels du bois, des chercheurs, des banques multilatérales de développement, des fonds d'investissements privés et des fondations philanthropiques. Ses quatre missions sont : promouvoir la valeur des forêts pour la société ; promouvoir la gestion durable et la conservation de la biodiversité en créant de la valeur au travers des outils de marché ; encourager des projets innovants et des acteurs économiques pour développer de tels marchés ; améliorer le bien-être des populations vivant dans ou à proximité des forêts (note du traducteur).
} 


\section{BIBLIOGRAPHIE}

BOSWORTH (D.), BROWN (H.). - Investing in the future: Ecological restoration and the U.S. Forest Service. - Journal of Forestry, vol. 105, $\mathrm{n}^{\circ}$ 4, 2007a, pp. 208-211.

BOSWORTH (D.), BROWN (H.). - After the timber wars: Collaborative community-based stewardship. Journal of Forestry, vol. 105, $\mathrm{n}^{\circ}$ 5, 2007b, pp. 271-273.

BRAND (D.). - Communication personnelle (avril 2004). Directeur du Global Forest Carbon Sequestration Program. - Sydney, Australie : Hancock Natural Resource Group.

BUTLER (B.J.) LEATHERBERRY (E.C.). - America's family forest owners. - Journal of Forestry, vol. 102, $\mathrm{n}^{\circ} 7$, 2004, pp. 4-9.

CARSON (R.). - Printemps silencieux. - Paris : Plon, [1962] 1963.

COLLINS (S.), BROWN (H.). - Ecological restoration calls for a new kind of language. - Ecological Restoration, vol. 26, $\mathrm{n}^{\circ} 3,2008$, pp. 213-218.

COLLINS (S.), LARRY (E.). - Caring for our natural assets: An ecosystem services perspective. In: Integrated Restoration of Forested Ecosystems To Achieve Multi-Resource Benefits / R.L. Deal, tech. ed.. Proceedings of the 2007 National Silviculture Workshop ; 7-11 mai ; Ketchikan, AK. - Portland, OR : U.S. Forest Service, Pacific Northwest Research Station, 2007. - Gen. Tech. Rep. PNW-733.

CORTNER (H.J.). - The governance environment: Linking science, citizens, and politics. In : Ecological restoration in southwestern ponderosa pine forests / P. Friederici, ed. - Washington, DC : Island Press, 2003. - pp. $70-80$.

GIBBONS (M.). - Science's new social contract with society. - Nature, 402(6761), 1999, pp. C81-C84.

GIPPERT (M.J.), DEWITTE (V.L.). - Forest plan implementation: Gateway to compliance with NFMA, NEPA, and other federal environmental laws. Critique of land management planning, vol. 10. FS-461. - Washington, DC : U.S. Forest Service, 1990. En ligne sur : http://www.fs.usda.gov/Internet/FSE_DOCUMENTS/stelprdb 5172352.pdf, consulté en mai 2011.

GROVES (R.C.), KUTNER (L.S.), STOMS (D.M.) [et al.]. - Owning up to our responsibilities: Who owns lands most important for biodiversity? In: Precious heritage: The status of biodiversity in the United States / B.A. Stein, L.S. Kutner, J.S. Adams, eds. - Londres : Oxford University Press, 2000. - pp. 275-300.

LEOPOLD (A.). - Conservation. In: Round river: From the journals of Aldo Leopold / L.B. Leopold, ed. London, UK : Oxford University Press, 1949. - pp. 145-157.

LEOPOLD (A.). - Deer and Dauerwald in Germany II: Ecology and policy. - Journal of Forestry, vol. 34, $\mathrm{n}^{\circ}$ 5, 1936, pp. 460-466.

LEOPOLD (A.). - Engineering and conservation. In: The River of the Mother of God and other essays by Aldo Leopold / S.L. Flader, J.B. Callicott, eds. - Madison, WI : University of Wisconsin Press, 1938. - pp. 249254.

LEOPOLD (A.). - Manuscrit inédit. - Cité dans : The essential Aldo Leopold: Quotations and commentaries / C. Meine, R.L. Knight, eds. - Madison, WI : University of Wisconsin Press, 1935. - 161 p.

LEOPOLD (A.). - Thinking like a mountain. In: A Sand County almanac and sketches here and there. London, UK : Oxford University Press, 1949. - pp. 129-133.

MEA (MILLENNIUM ECOSYSTEM ASSESSMENT). - Ecosystems and human well-being: Synthesis. Washington, DC : Island Press, 2005. En ligne sur : http://www.millenniumassessment.org, consulté en juin 2009.

SMITH (N.), DEAL (R.), KLINE (J.), BLAHNA (D.), PATTERSON (T.), SPIES (T.A.), BENNETT (K.). - Ecosystem services as a framework for forest stewardship: Deschutes National Forest overview. - Portland, OR : U.S. Department of Agriculture, Forest Service, Pacific Northwest Research Station, 2011. - 46 p. - Gen. Tech. Rep. PNW-852.

SMITH (W.B.) MILES (P.D.) VISSAGE (J.S.), PUGH (S.A.). - Forest resources of the United States, 2002. St. Paul, MN : USDA Forest Service, North Central Research Station, 2004. - Gen. Tech. Rep. GTR-NC-241.

STEIN (S.M.), McROBERTS (R.E.), MAHAL (L.G.), CARR (M.A.), ALIG (R.J.), COMAS (S.J.), THEOBALD (D.M.). Private forests, public benefits: Housing development and other pressures on private forest contributions. Projet. - Portland, OR : USDA Forest Service, Pacific Northwest Research Station, 2009. - 34 p.

USDA Forest Service. - National Forest System land and resource management planning. Sec. 219.19 : Fish and wildlife resource. - Washington, DC : USDA Forest Service, 1982. En ligne sur : http://www.fs.fed .us/emc/nfma/includes/nfmareg.html\#Diversity, consulté en avril 2011. 
USDA Forest Service. - National Forest System land management planning: Proposed rule. 36 CFR part 219. - Federal Register 76(30), 2011, pp. 8480-8528. En ligne sur : https://fs.usda.gov/Internet /FSE_DOCUMENTS/stelprdb5270250.pdf, consulté en avril 2011.

USDA Forest Service. - National roadmap for responding to climate change. - Washington, DC : USDA Forest Service, 2010. En ligne sur : http://www.fs.fed.us/climatechange/pdf/roadmap.pdf, consulté en avril 2011.

USDA Forest Service. - The principal laws relating to Forest Service activities. National Forest Management Act, sec. 6(g)(3)(B). - Washington, DC : Government Printing Office, 1993.

\section{L'US FOREST SERUICE ET LA BIODIUERSITE [Résumé]}

L'US Forest Service a été créé en 1905 pour remédier à une déforestation à grande échelle et à la dégradation des bassins versants aux États-Unis d'Amérique. Dans un premier temps, la protection de la faune sauvage s'est focalisée sur celle du gibier, et tout particulièrement sur le contrôle des prédateurs. Après la Seconde Guerre mondiale, l'attention prioritaire de l'US Forest Service se déplaça vers la production et la récolte de bois, afin de satisfaire une demande en bois de construction en forte croissance. De nouvelles lois conçues pour trouver un équilibre entre les multiples usages de la forêt et des terrains pastoraux échouèrent et furent à l'origine d'un conflit entre les intérêts liés au bois et ceux liés à la biodiversité. Dans les années 1990, à la suite du conflit de la Chouette tachetée, l'US Forest Service abandonna son approche traditionnelle en termes de produits au profit d'une gestion des forêts et des terrains pastoraux en quête de résultats sur le long terme tels que des écosystèmes en bonne santé et résilients à l'échelle du paysage, prenant en compte la protection de la biodiversité. Quoi qu'il en soit, aujourd'hui le contexte du changement climatique, de l'artificialisation des sols et d'autres questions émergentes exige de renoncer à l'approche traditionnelle de la protection des espèces, prises une à une, dans des espaces protégés, pour prôner une approche en termes de services écosystémiques sur tous les territoires, publics et privés. L'US Forest Service continuera à investir dans le "capital Nature " sur les terrains publics en vue de fournir des biens et des services d'intérêt public. Avec les restrictions budgétaires, l'Agence a commencé à explorer de nouvelles sources de revenus pour les propriétaires privés, venant de capitaux privés par le biais des marchés environnementaux portant sur la biodiversité, le carbone, les zones humides et l'eau.

\section{BIODIUERSITY AND THE U.S. FOREST SERUICE [Abstract]}

The U.S. Forest Service was established in 1905 to address devastating environmental problems on U.S. range and forest lands. Unlike its sister agency, the National Park Service, the Forest Service was established with a "multiple-use" mission. That is, rather than to protect and preserve natural resources within the public forest domain, the Forest Service's mission was to allow grazing, mining, and timber production, as long as these uses could be conducted sustainably. The spotted owl issue in the Pacific Northwestern US shifted forever the policy framework for managing the public forests, as litigation challenged the agencies' ability to continue harvesting old growth timber and protect the owl. The harvesting of old growth timber virtually ceased on national forests. Changes followed quickly in many sectors, including forest education and certification, rules for forest management at both the state and national level, and timber industry standards of practice as these uses could be conducted sustainably. Thus, moving from three decades of intensively managing natural forests, the Forest Service has shifted priorities to protecting watersheds and biodiversity, reducing the risk of wild fire, and adapting to climate change. This has required new skills, tools, and policies, including a new forest planning framework. With budgets contracting, the Forest Service has begun exploring new sources of revenue, including private capital from environmental markets for biodiversity, carbon, wetlands, and water. 